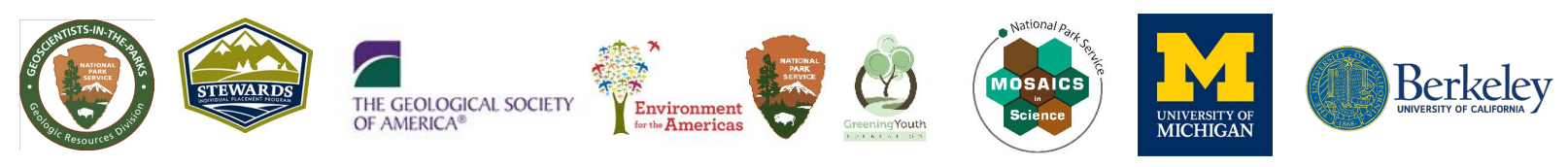

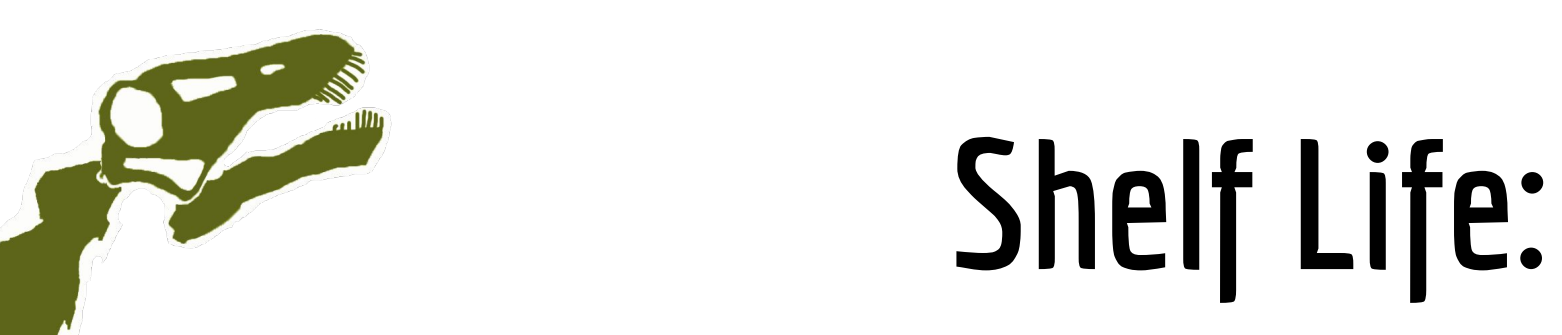

\title{
Updating and Preserving Collections at Dinosaur National Monument
}

\author{
Kellyn McKnight, Tut Tran, \\ Julia Anderson, ReBecca Hunt-Foster
}
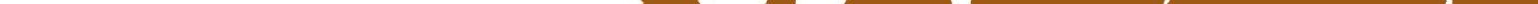


\section{Goals}

- Organize collection spaces and mitigate threats to specimens

- Catalog specimens to improve record-keeping at Dinosaur National Monument

- Stabilize unsupported and/or deteriorating fossils

- Spread awareness of fossil conservation efforts through public outreach 


\section{Quarry Exhibit Hall}

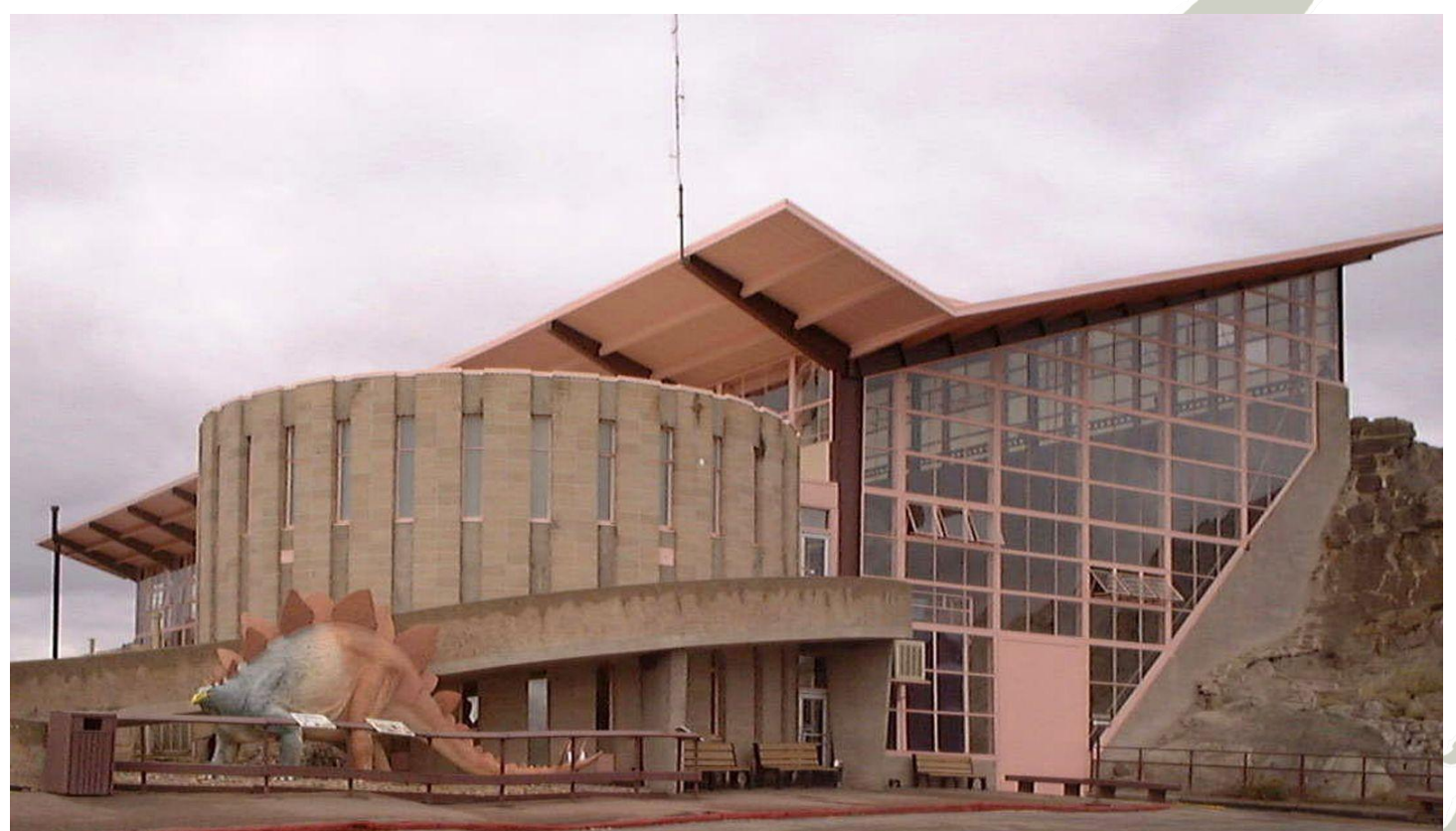

https://www.flaminggorgecountry.com/Museums 


\section{Quarry Exhibit Hall}

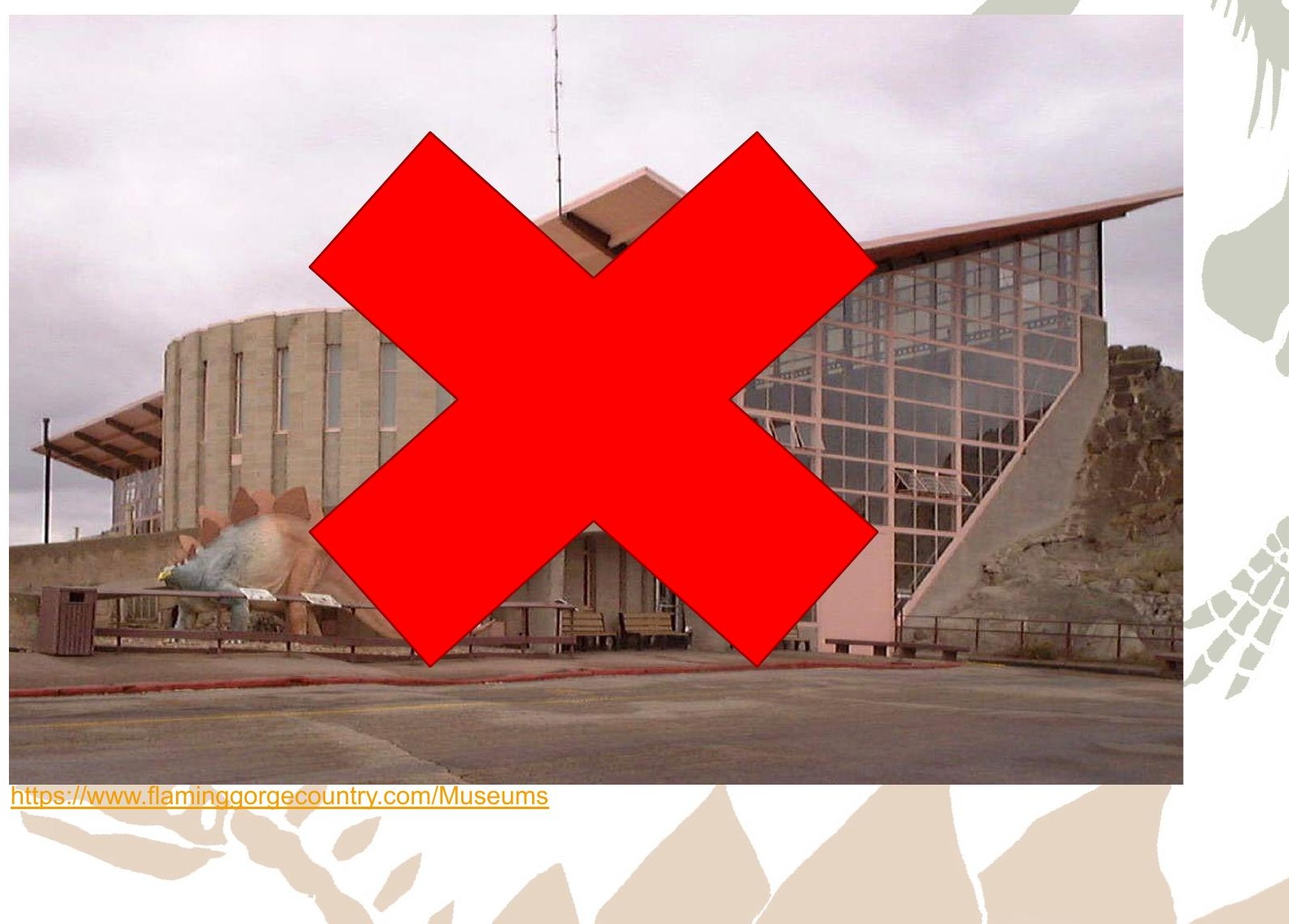




\section{Quarry Exhibit Hall 2.0}

- Quarry Exhibit Hall included collections facility until 2006

- $\quad$ Facility was removed when QEH waS condemned

- Specimens formerly housed there had to be moved quickly

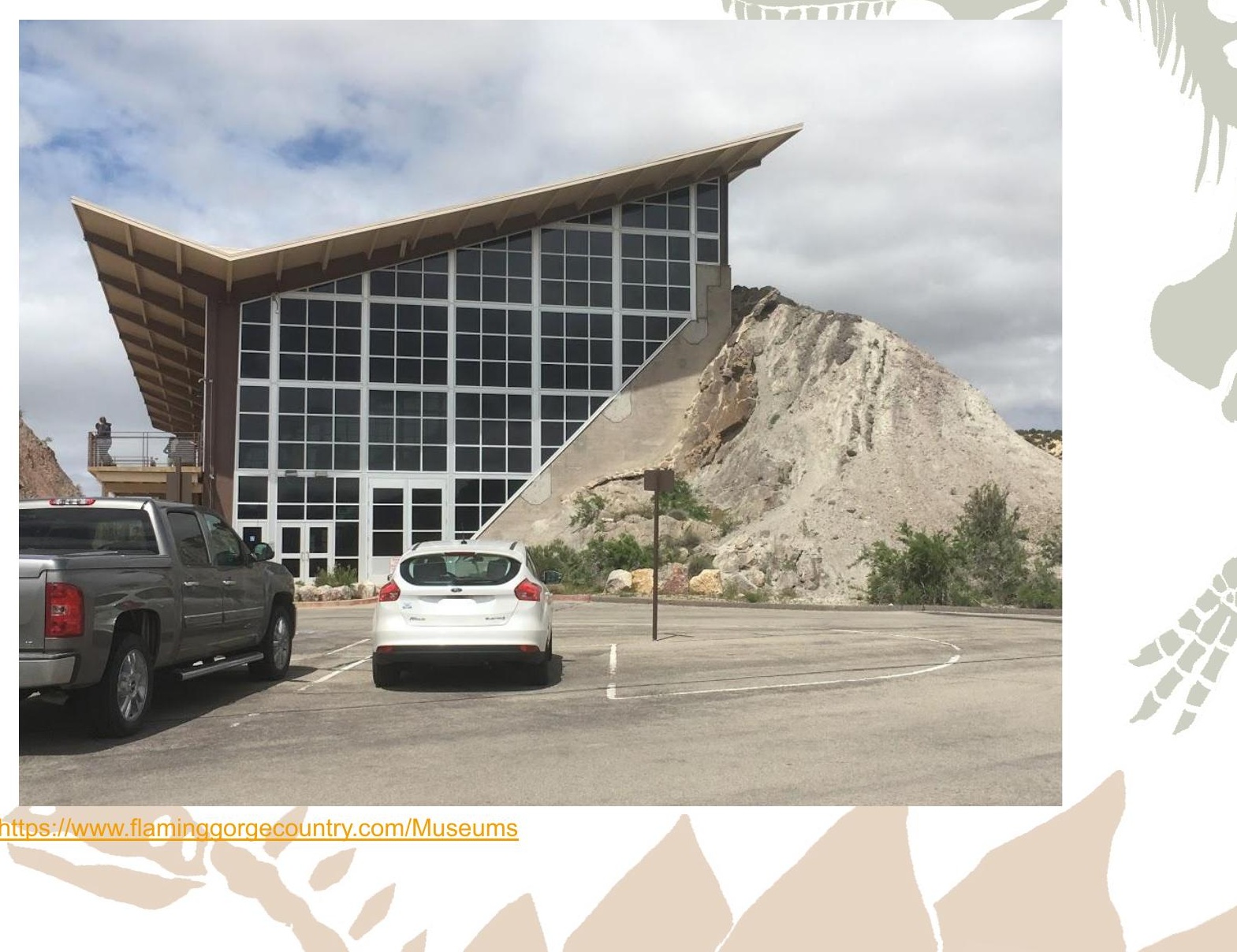




\section{Collections Facilities}

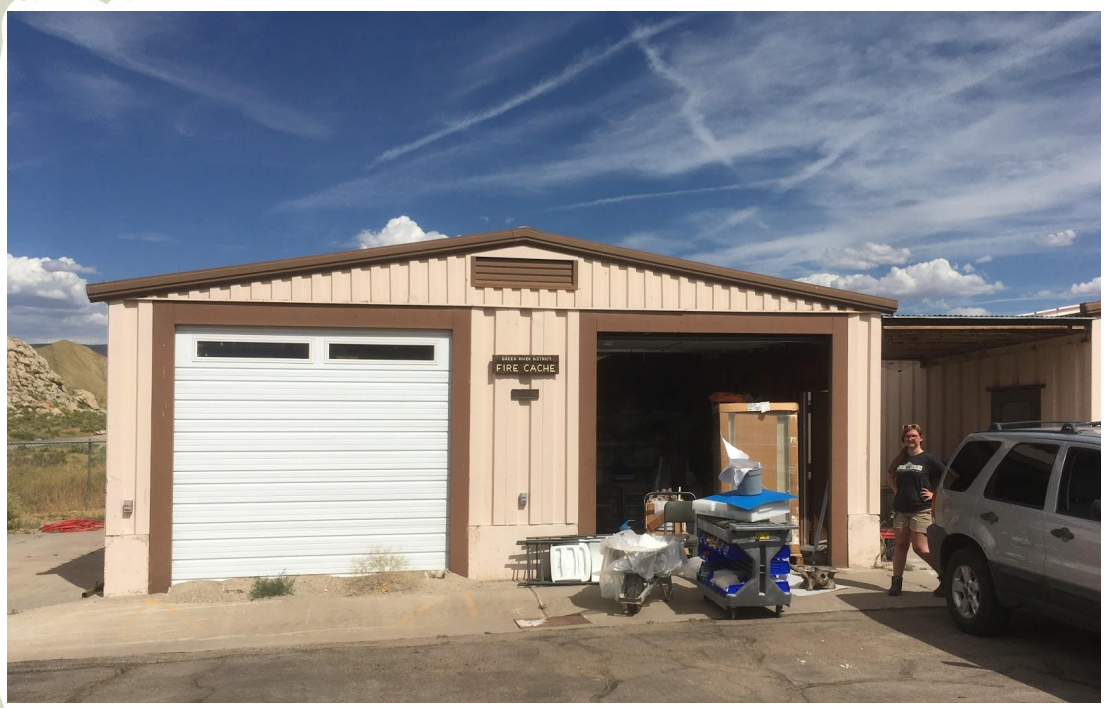

Fire Cache

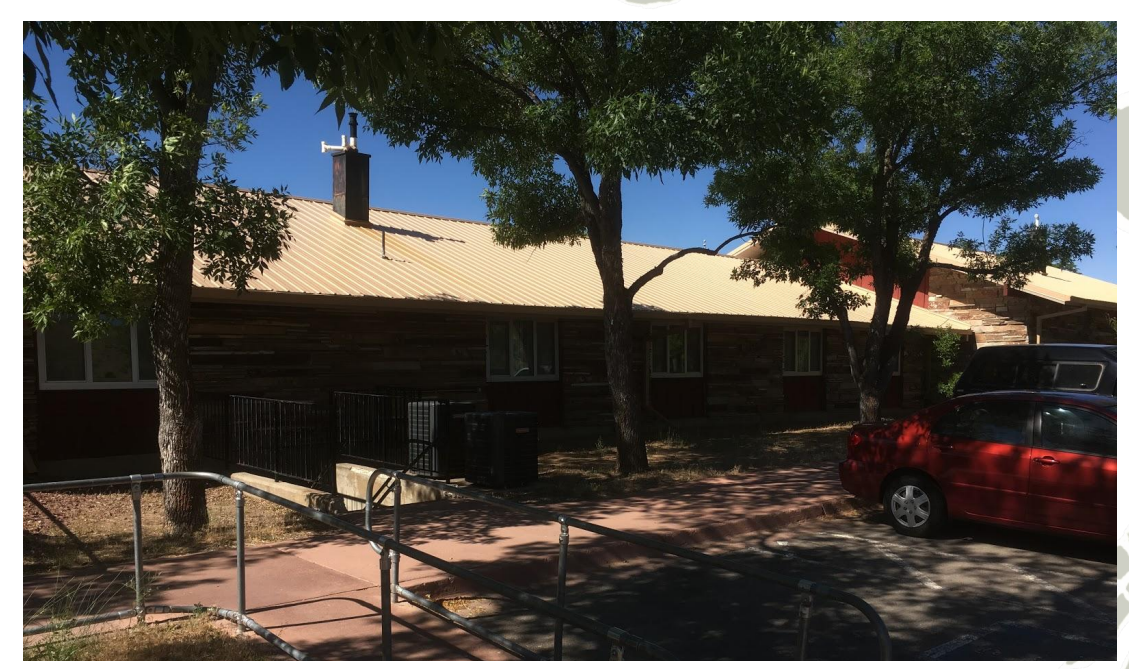

Canyon Visitor Center

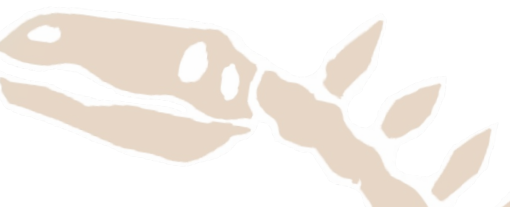


Collections Facilities: Fire Cache (Utah)

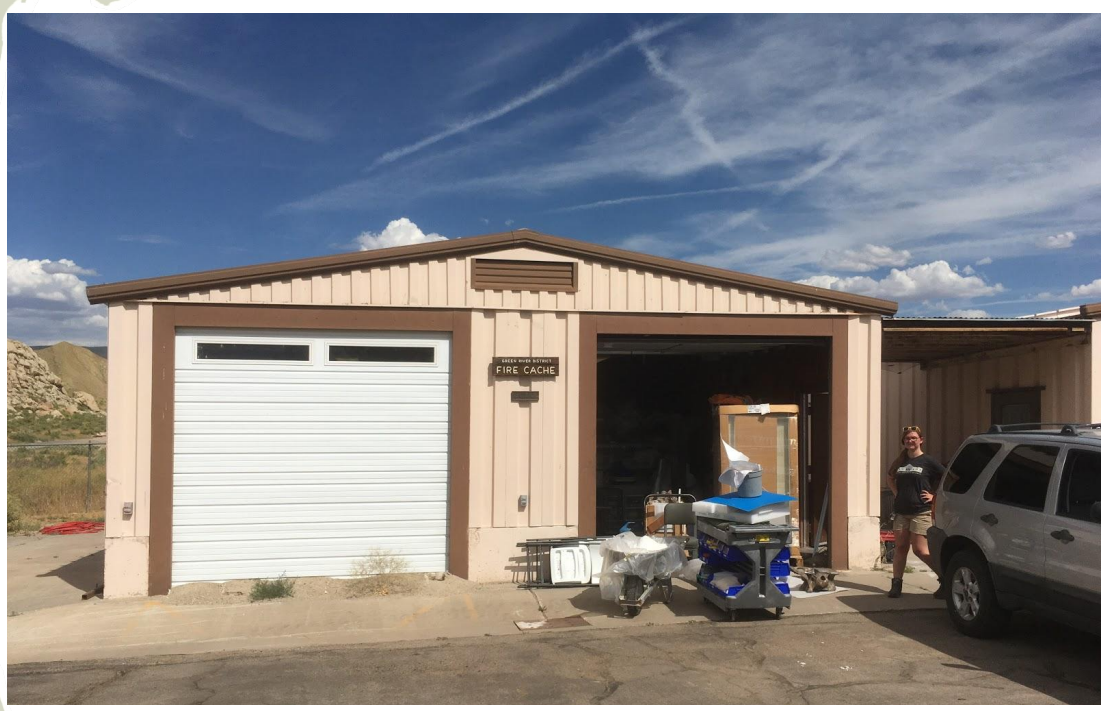

- Temperature Fluctuation

- Rainwater

- Insects

Fire Cache 
Collections Facilities: Fire Cache (Utah)

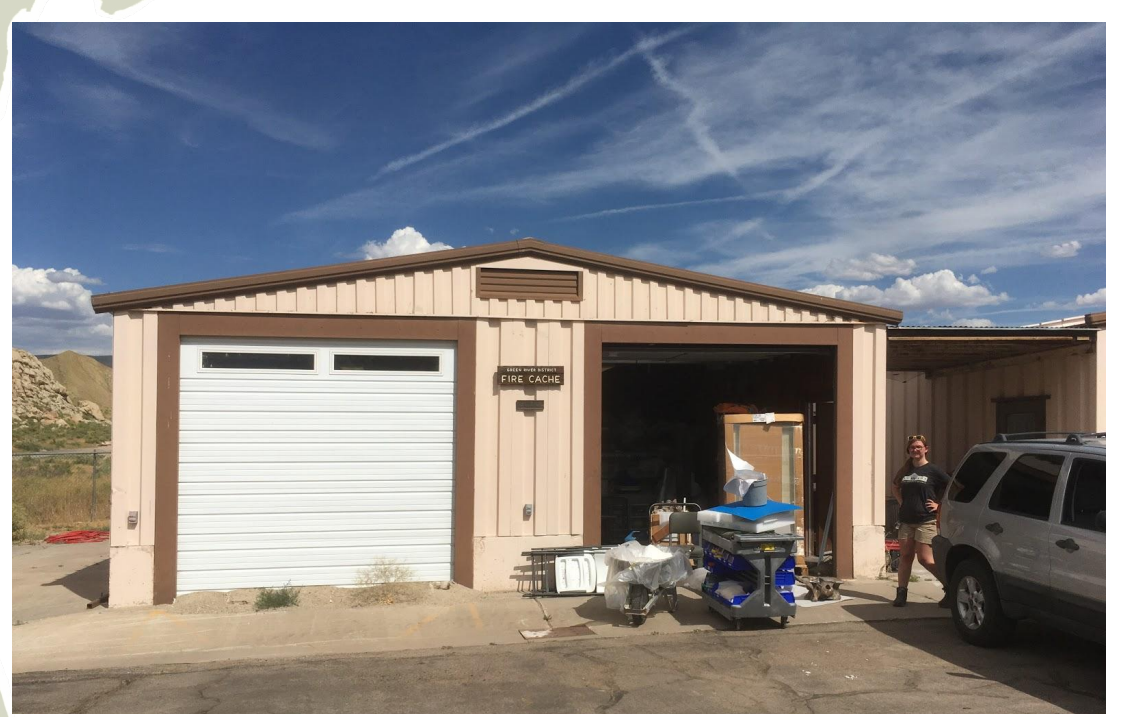

Fire Cache

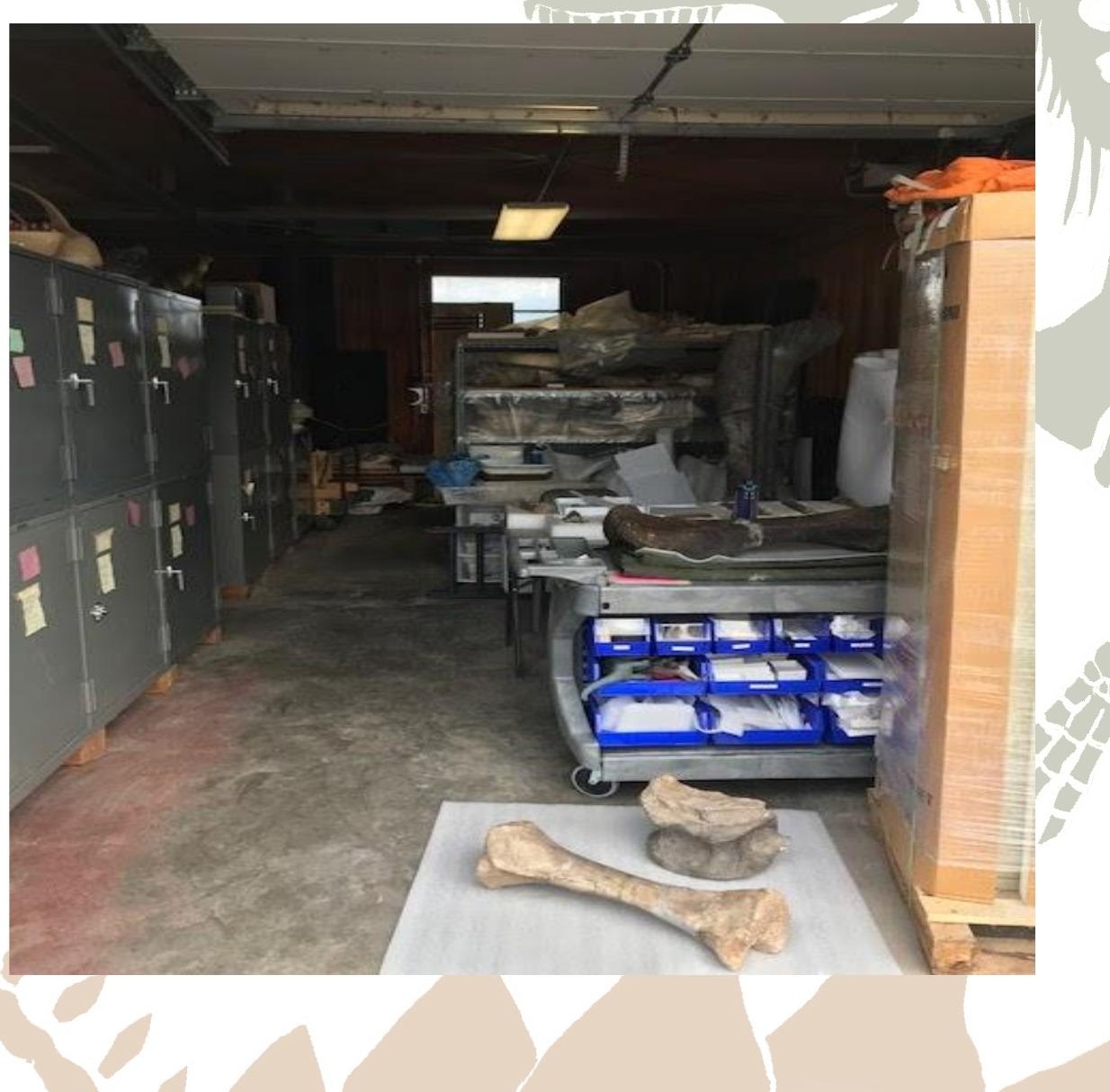


Collections Facilities: Fire Cache (Utah)

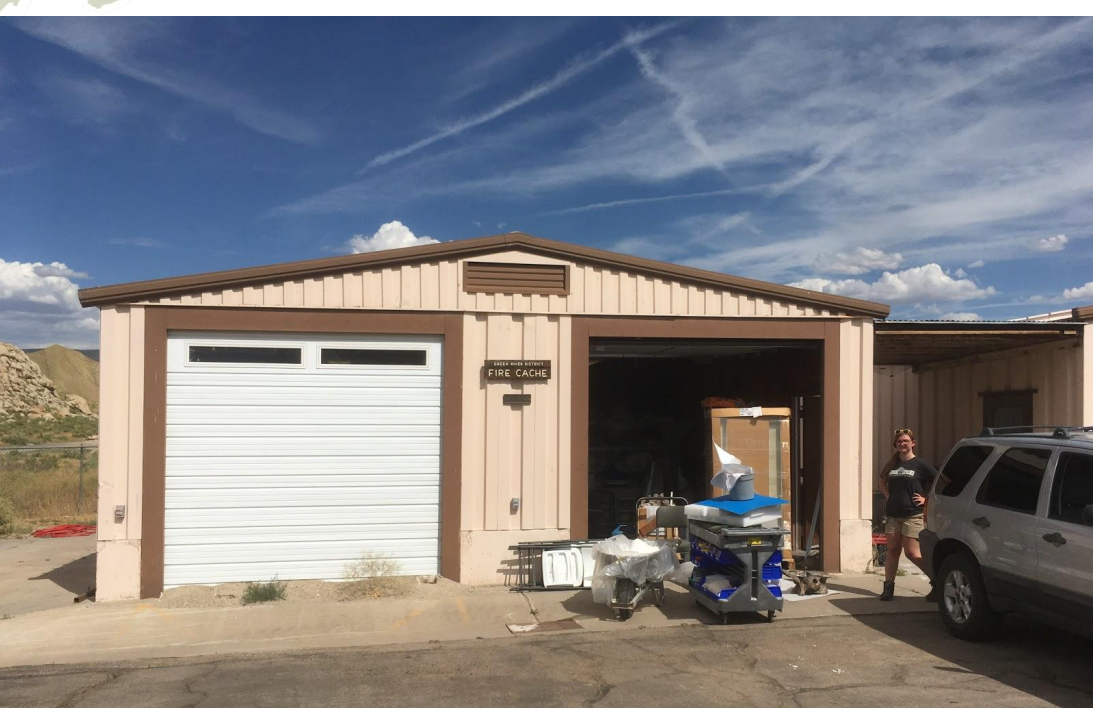

Fire Cache

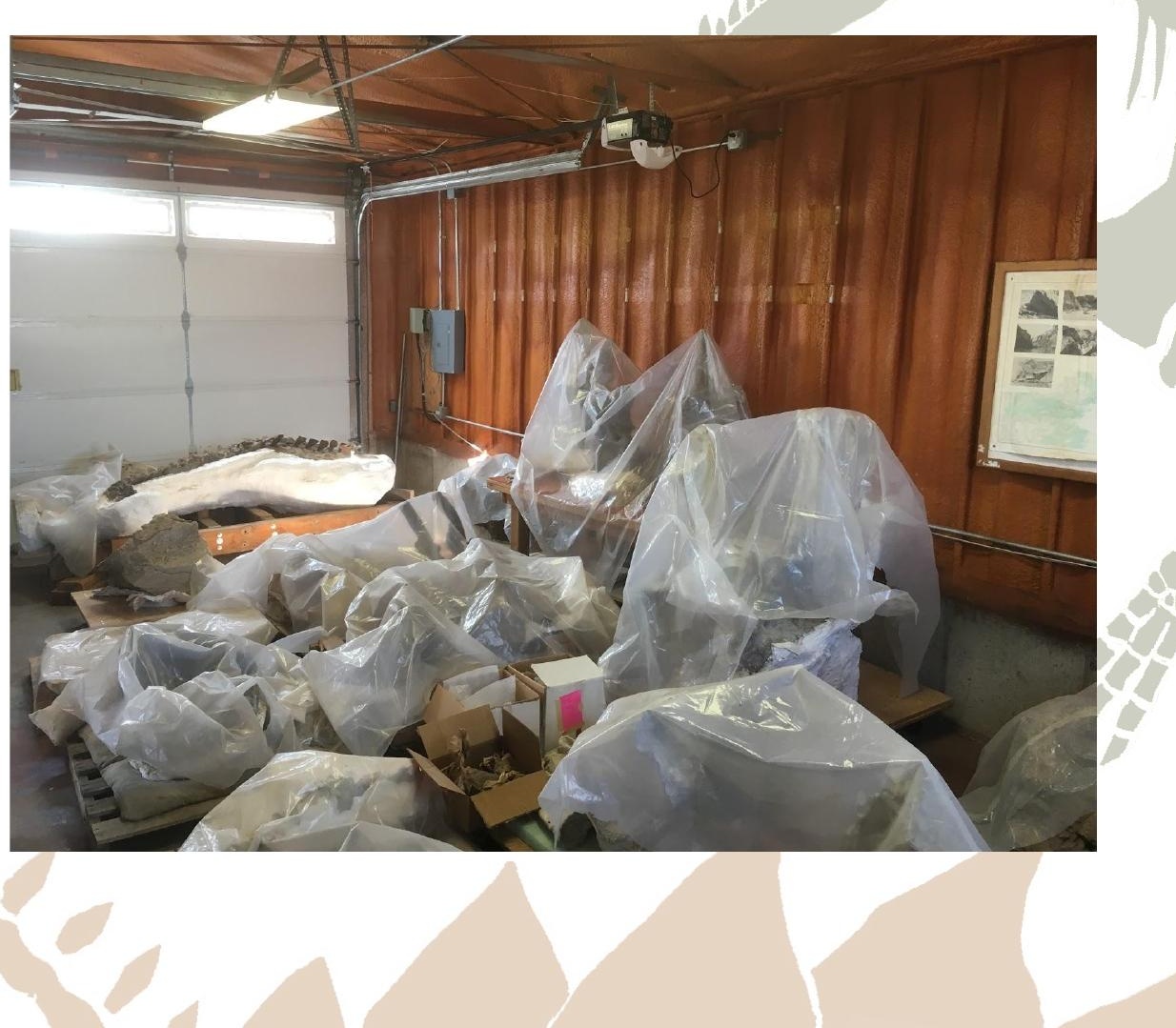




\section{Collections Facilities: Canyon Visitor Center (Colorado)}

- Holds paleontology and archeology collections

○ Uncatalogued

- Rodents

○ Hantavirus

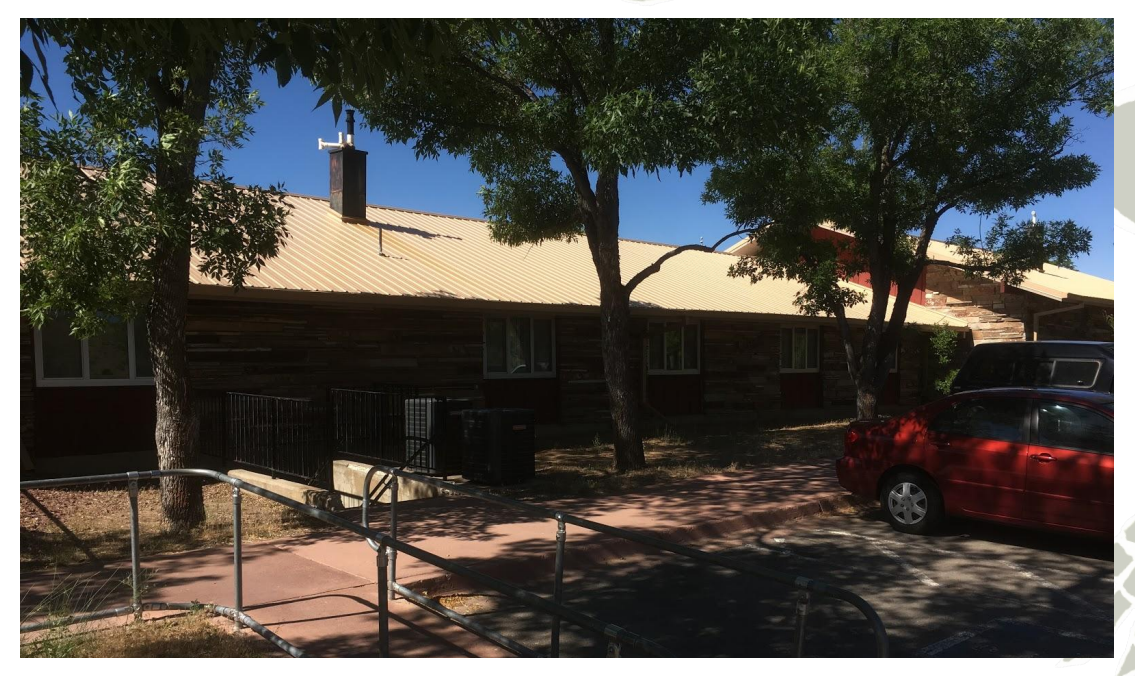

Canyon Visitor Center 
Collections Facilities: Canyon Visitor Center (Colorado)
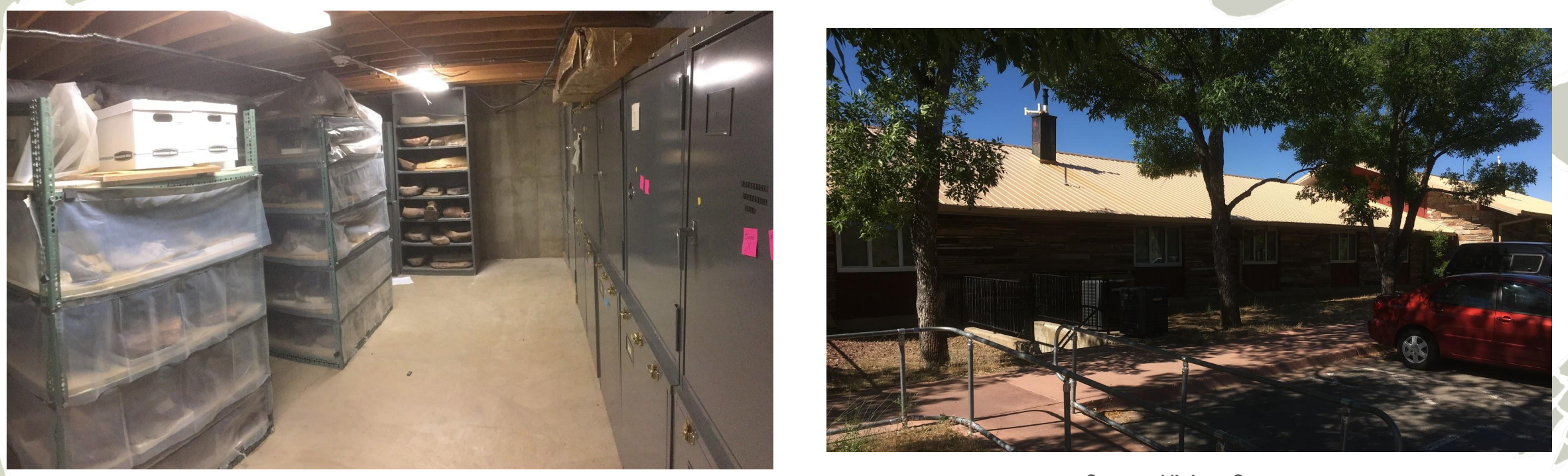

Canyon Visitor Center

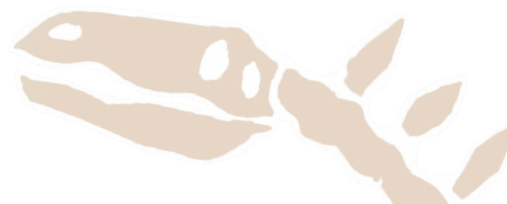


Collections Facilities: Canyon Visitor Center (Colorado)
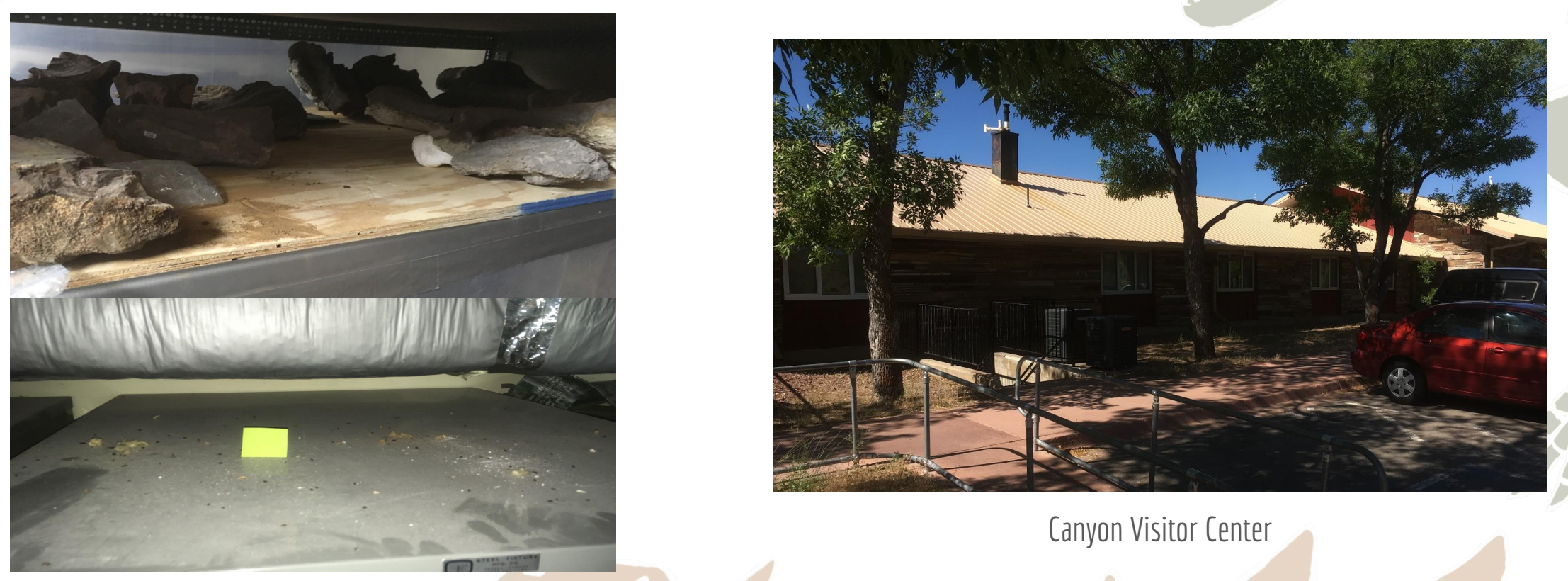

Canyon Visitor Center 


\section{Specimen Conditions}
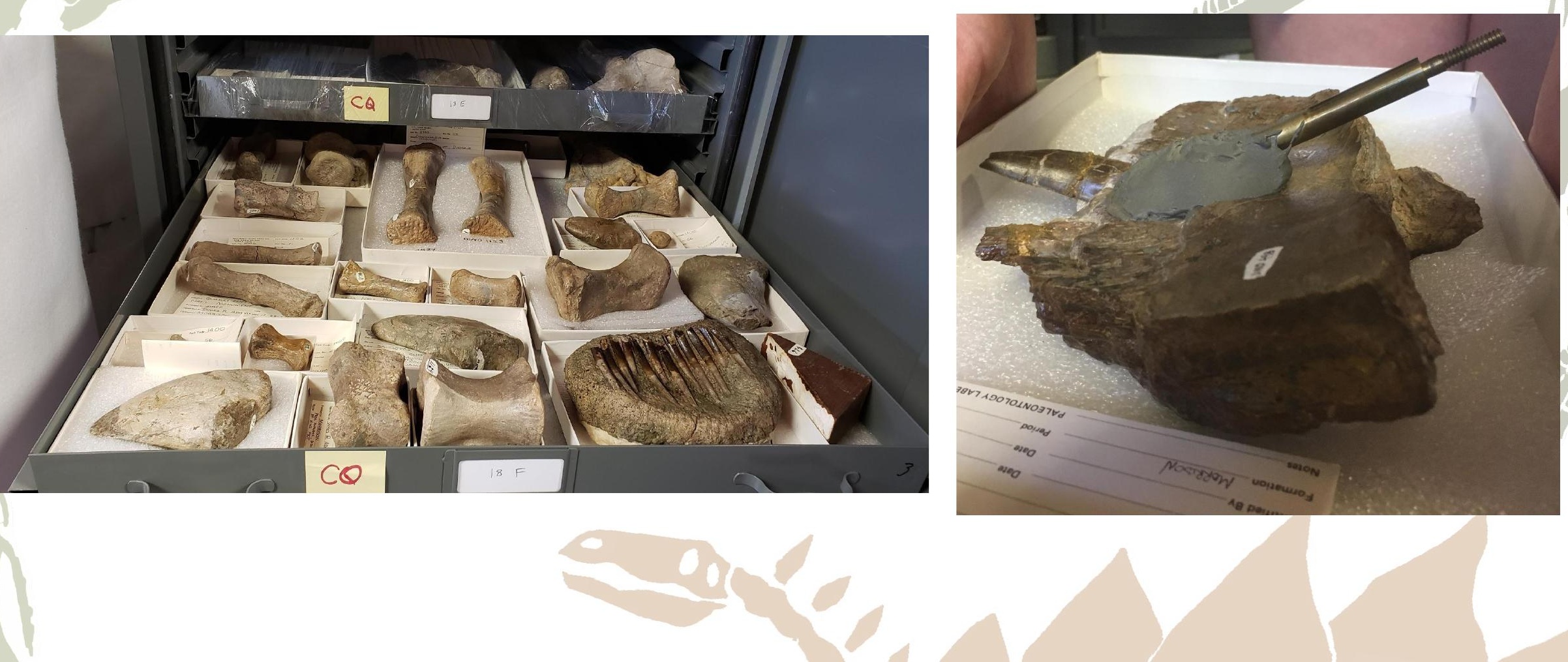


\section{Specimen Conditions}

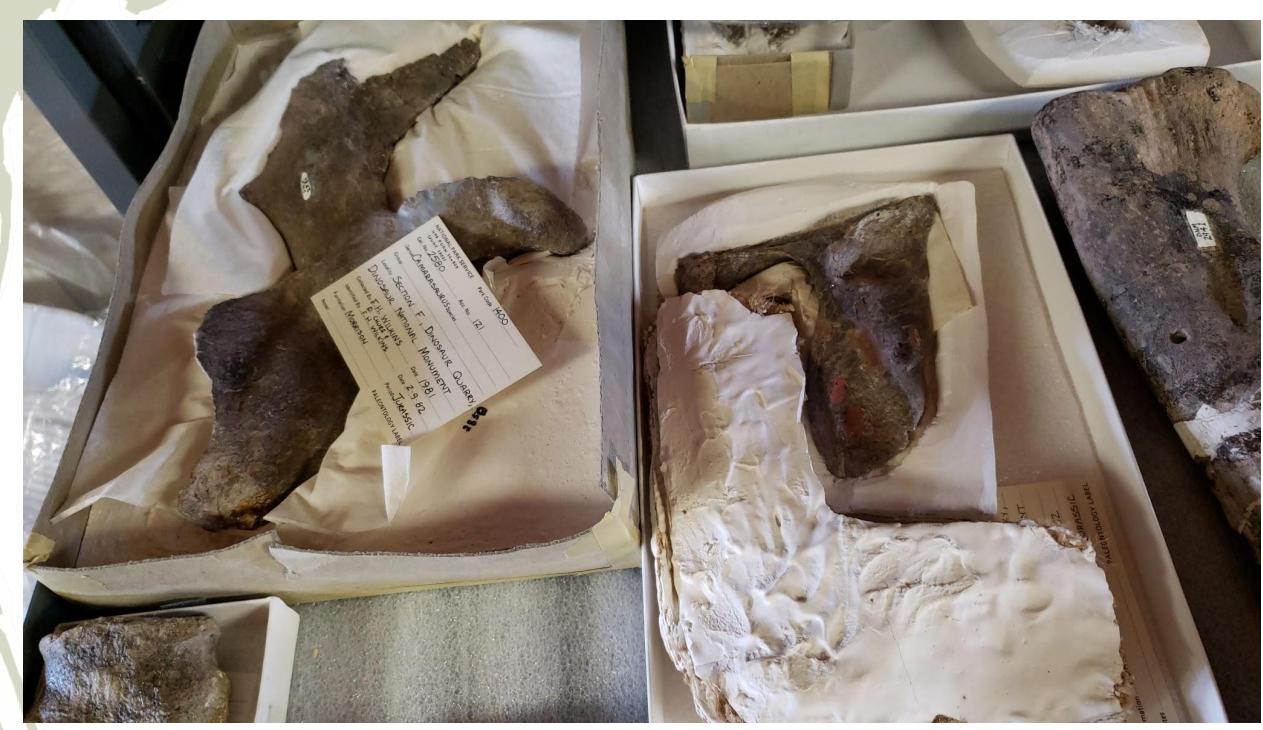




\section{Specimen Conditions}

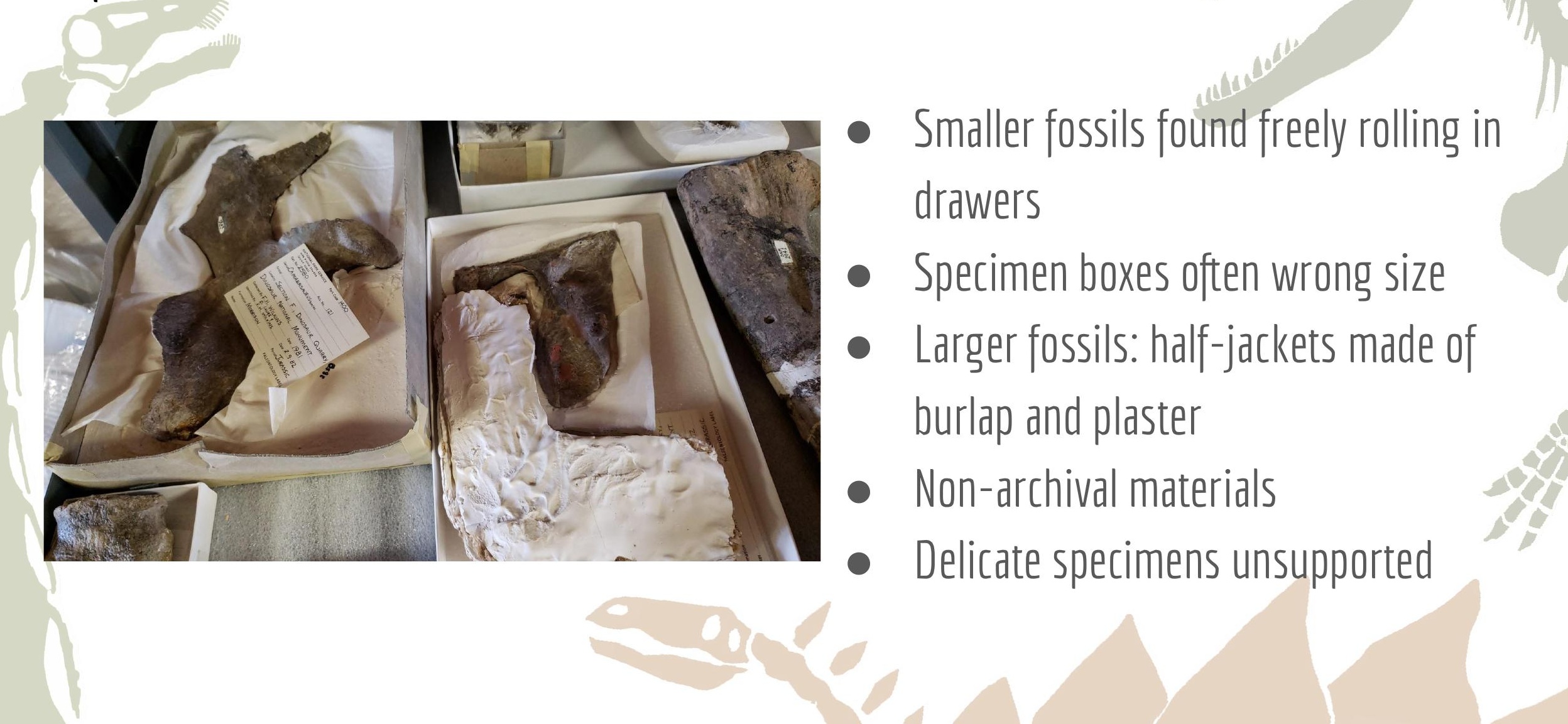




\section{Tackling the Issues}

- Hantavirus Mitigation

- Catalog and Inventory

- Plaster Cradle Construction

- Foam Cradle Construction

- Outreach E Education 


\section{Tackling the Issues}

\section{- Hantavirus Mitigation}

- Catalog and Inventory

- Plaster Cradle Construction

- Foam Cradle Construction

- Outreach \& Education 


\section{Tackling the Issues}

\section{- Hantavirus Mitigation:}

\section{Hantavirus}

- Found in rodent feces and urine

- Can contract Hantavirus Pulmonary Syndrome (HPS) by inhalation or touch

- HPS is rare, but lethal. No vaccine or cure. 


\section{Tackling the Issues}

\section{- Hantavirus Mitigation:}

\section{PPE}

- 3M Tyvek body suit

- 3M half-face respirator

- Nitrile gloves

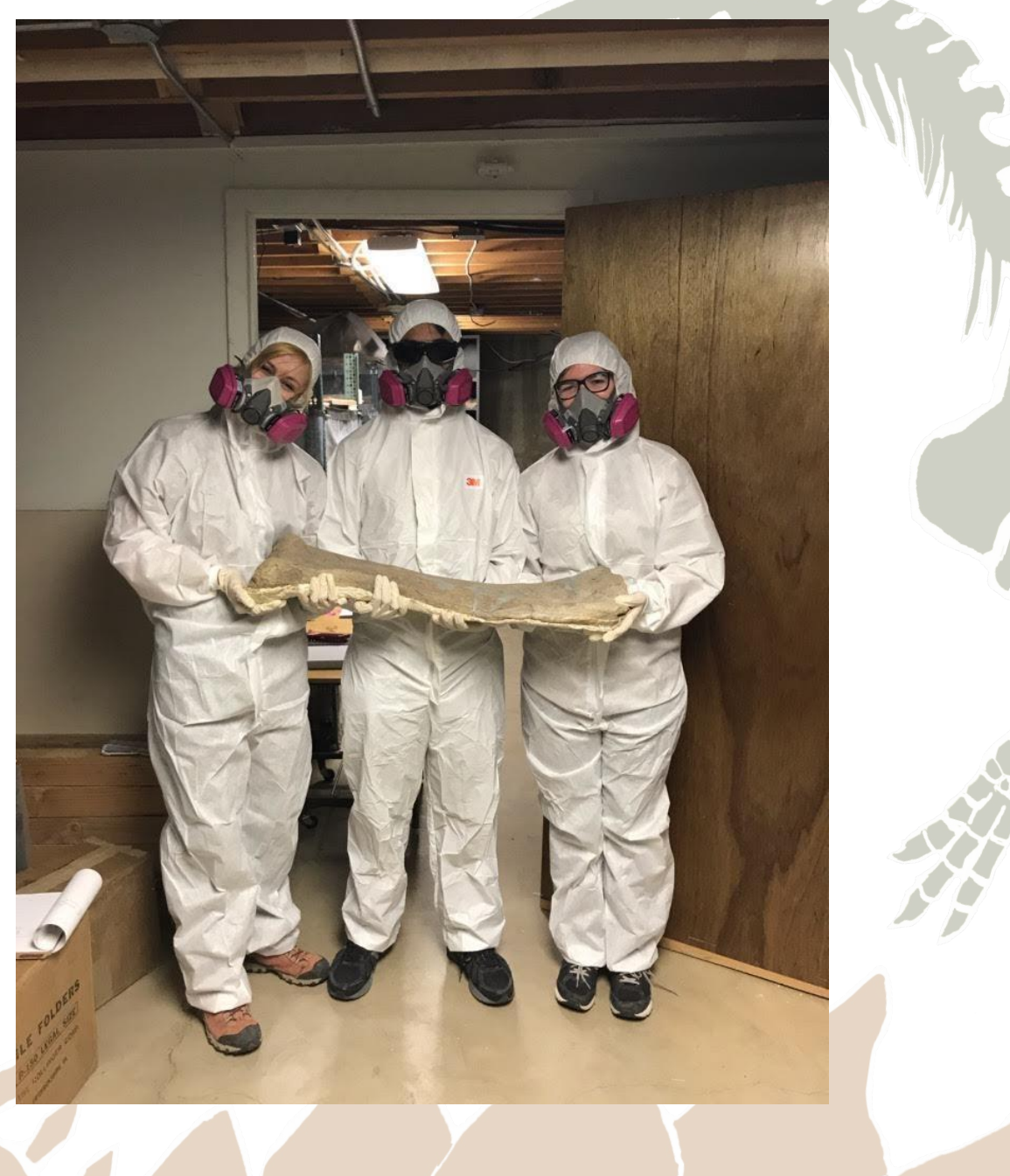




\section{Tackling the Issues}

\section{- Hantavirus Mitigation:}

\section{Cleaning Equipment}

- Bleach solution

- Mouse repellent

- Steel wool 


\section{Tackling the Issues}

\section{- Hantavirus Mitigation:}

\section{Decontamination}

- Sun bleaching for min. of 4 hours

- Spraying the worst areas with bleach solution

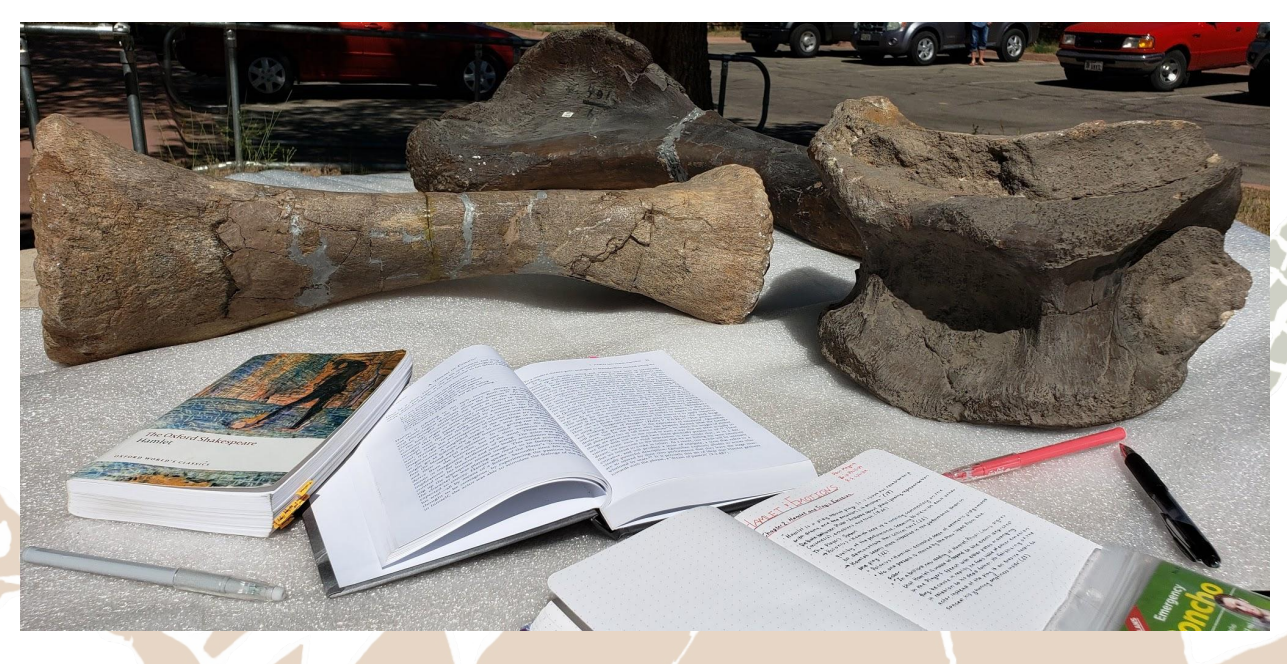




\section{Tackling the Issues}

- Catalog and Inventory

○ Filled in digital catalog of paleo, archeo, and herbarium collections

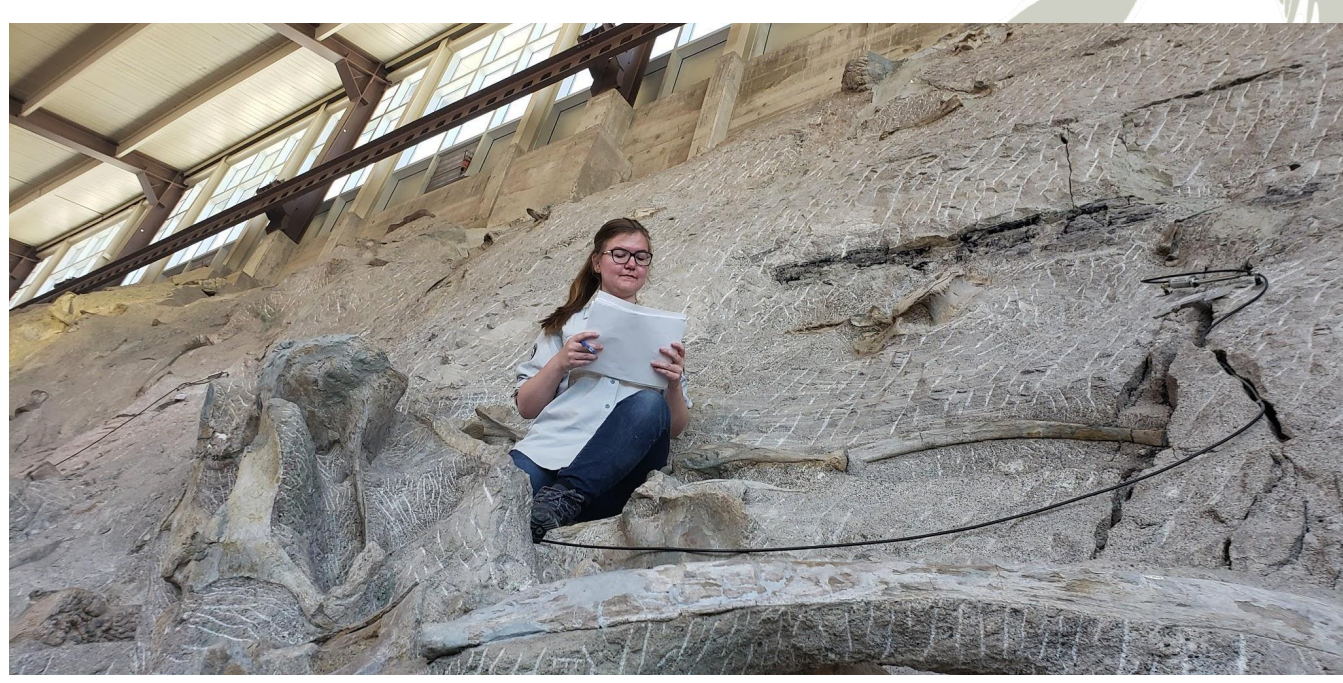

- Spreadsheet should contain info on 43-44,000+ specimens when complete

- Physical inventory 


\section{Tackling the Issues}

- Hantavirus Mitigation

- Catalog and Inventory

- Plaster Cradle Construction

- Foam Cradle Construction

- Outreach \& Education 


\section{Tackling the Issues}

- Plaster Cradle Construction

- ${ }^{\frac{11}{4}}$ Ethafoam sheets / felt

- Fiberglass

- FGR 95 Hydrocal plaster

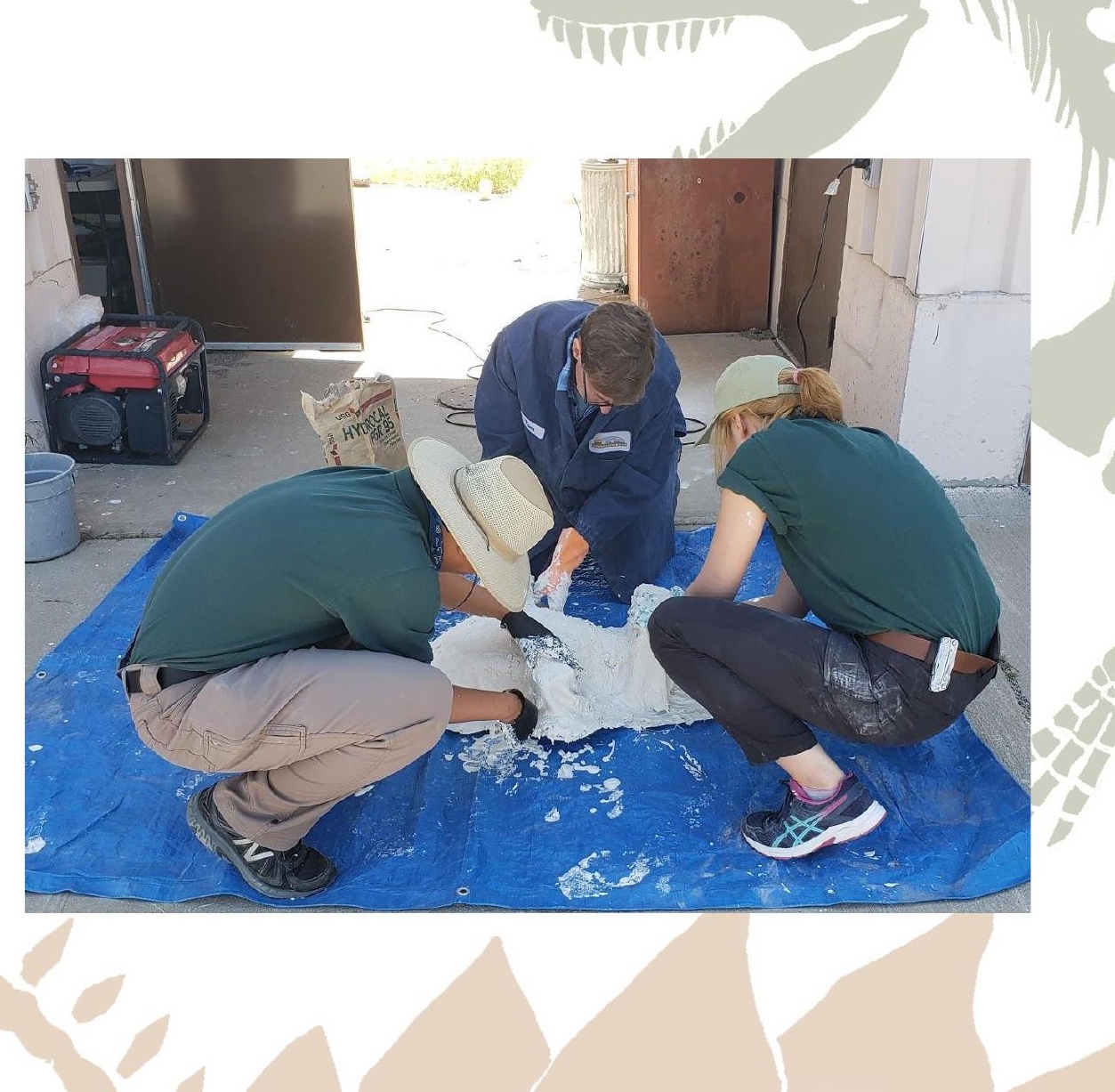




\section{Tackling the Issues}

- Plaster Cradle Construction

- ${ }^{\frac{11}{4}}$ Ethafoam sheets / felt

- Fiberglass

- FGR 95 Hydrocal plaster

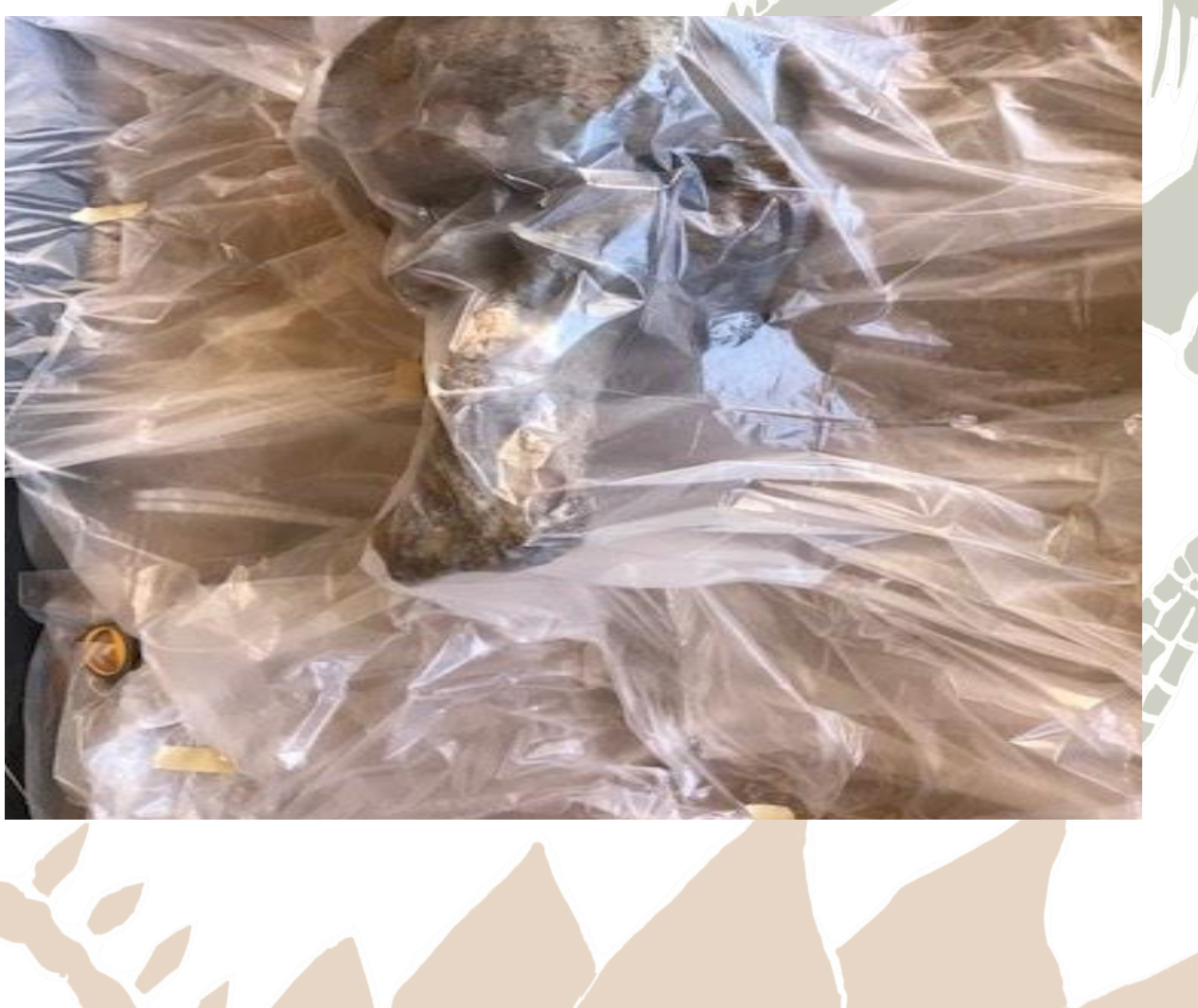




\section{Tackling the Issues}

- Plaster Cradle Construction

- ${ }^{\frac{11}{4}}$ Ethafoam sheets / felt

- Fiberglass

- FGR 95 Hydrocal plaster

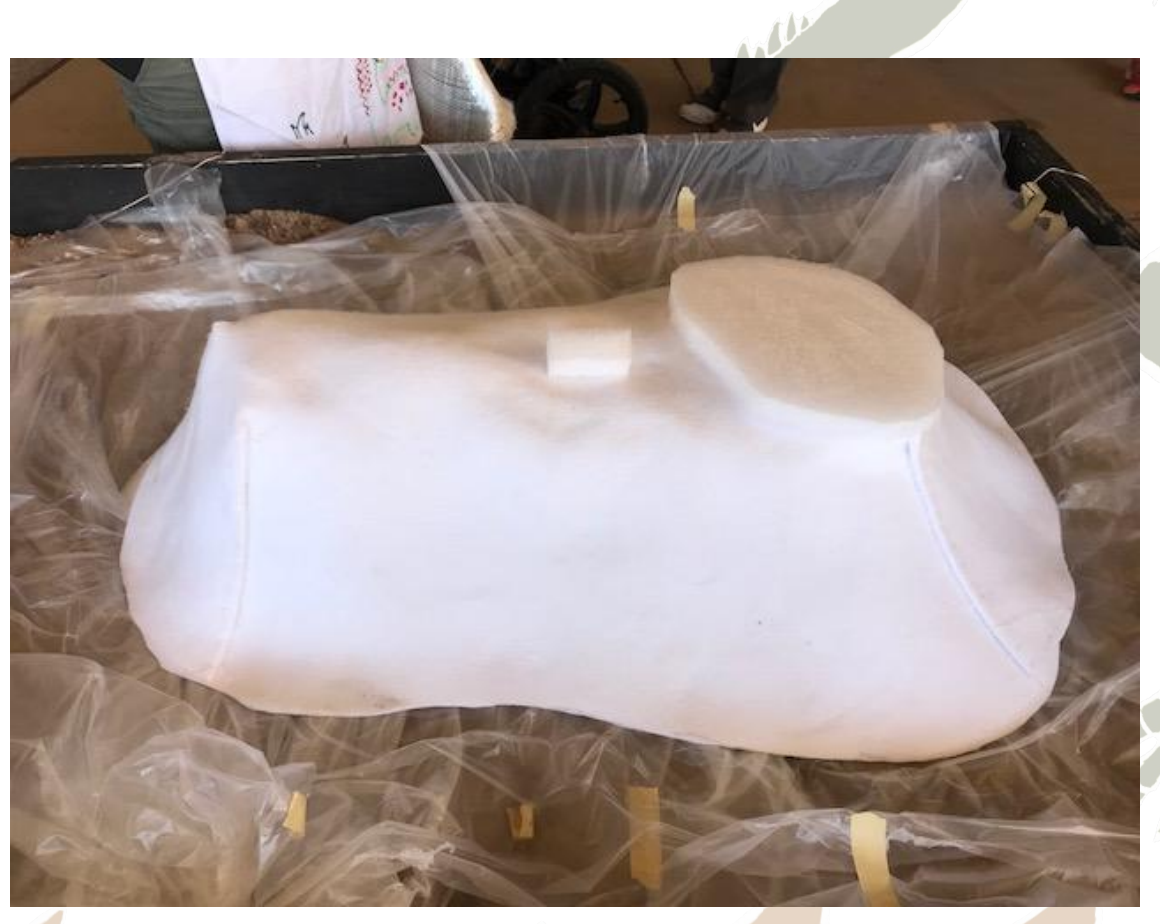




\section{Tackling the Issues}

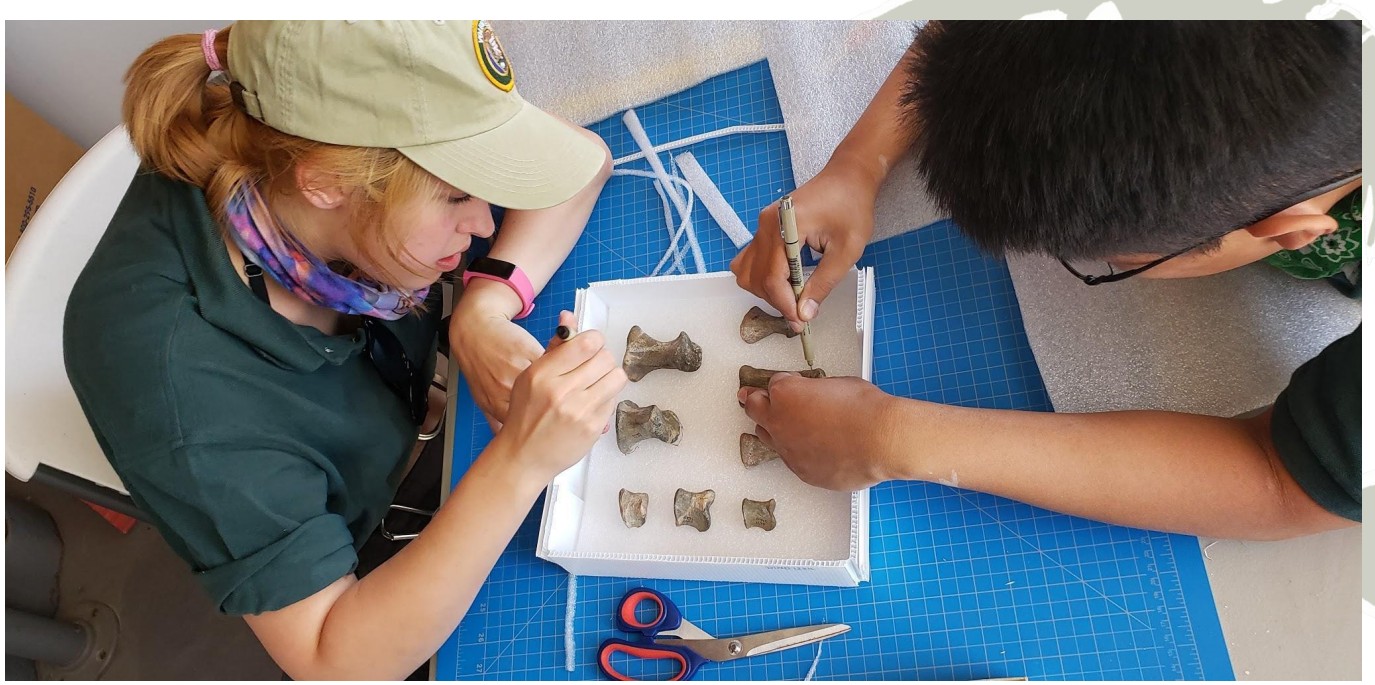

- Foam Cradle Construction

- $\frac{11}{4}$ Ethafoam sheets or blocks

- Tyvek paper

- B-72 adhesive (acryloid \& acetone)

- Coroplast (corrugated plastic)

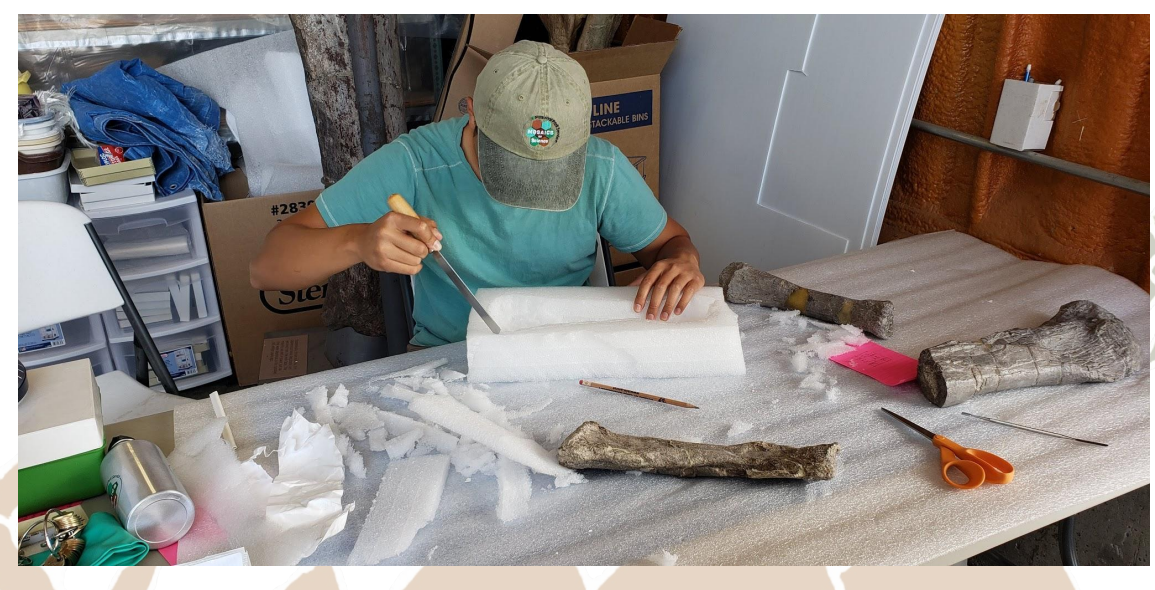




\section{Tackling the Issues}

- Foam Cradle Construction

- $\quad 1_{4}^{1 "}$ Ethafoam sheets or blocks

- Tyvek paper

- B-72 adhesive (acryloid \& acetone)

- Coroplast (corrugated plastic) 


\section{Tackling the Issues}

- Foam Cradle Construction

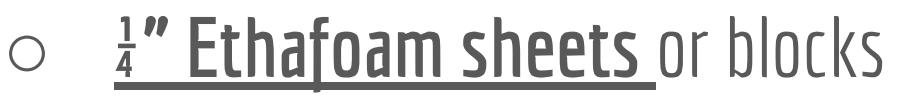

- Tyvek paper

- B-72 adhesive (acryloid \& acetone)

- Coroplast (corrugated plastic)
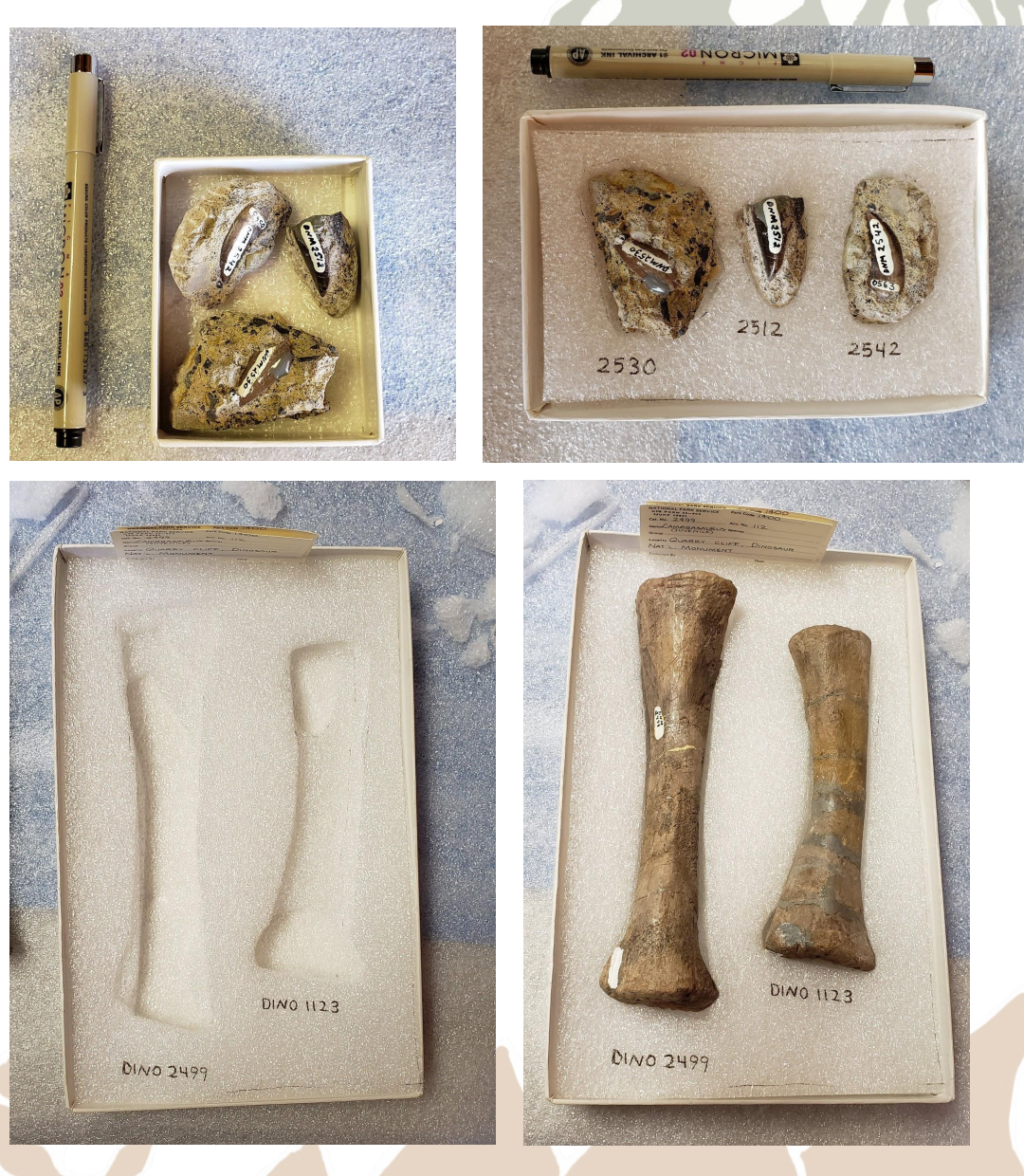
Tackling the Issues

- Foam Cradle Construction

- ${ }^{1 "}$ Ethafoam sheets or blocks

- Tyvek paper

- B-72 adhesive (acryloid \&s acetone)

- Coroplast (corrugated plastic) 


\section{Tackling the Issues}

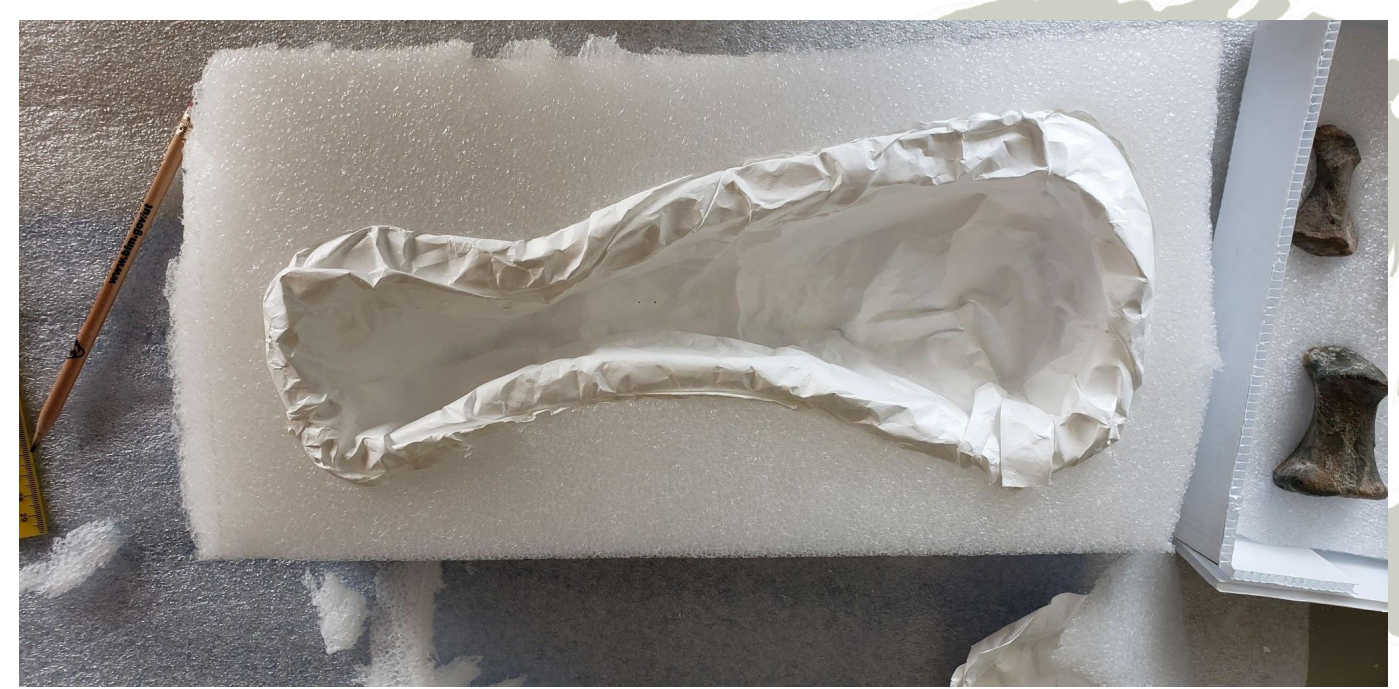

- Foam Cradle Construction

- $\underline{11}$ Ethafoam sheets or blocks

- Tyvek paper

- B-72 adhesive (acryloid \& acetone)

- Coroplast (corrugated plastic)

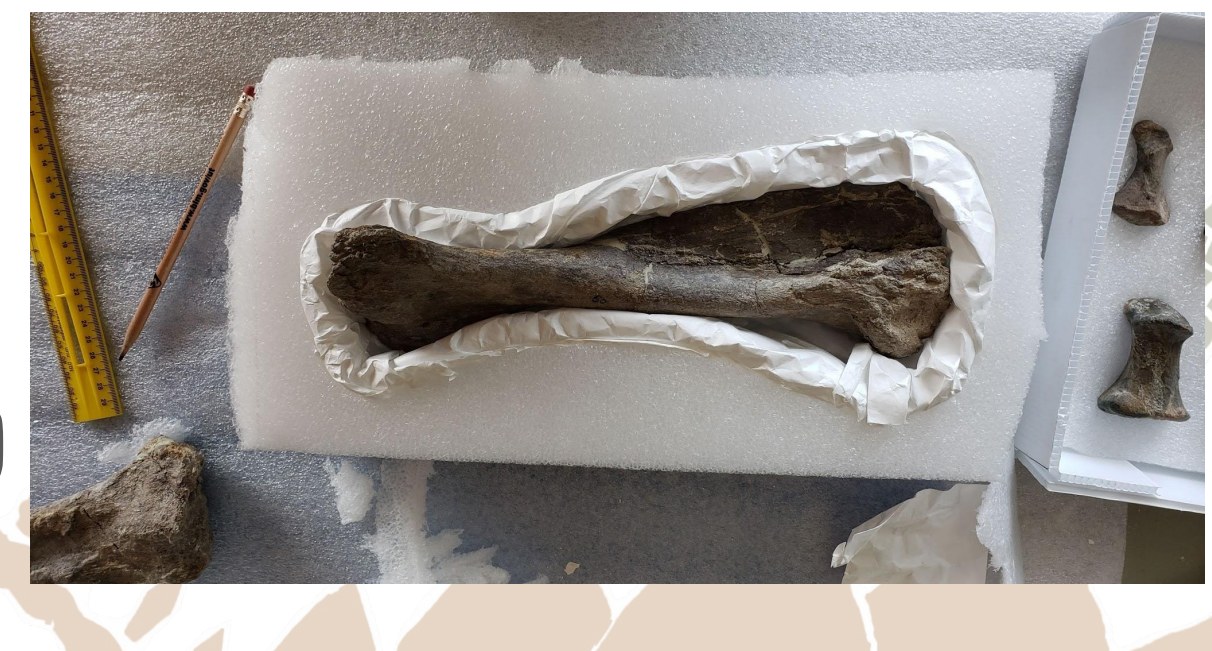




\section{Tackling the Issues}

- Hantavirus Mitigation

- Catalog and Inventory

- Plaster Cradle Construction

- Foam Cradle Construction

- Outreach \& Education 


\section{Tackling the Issues}

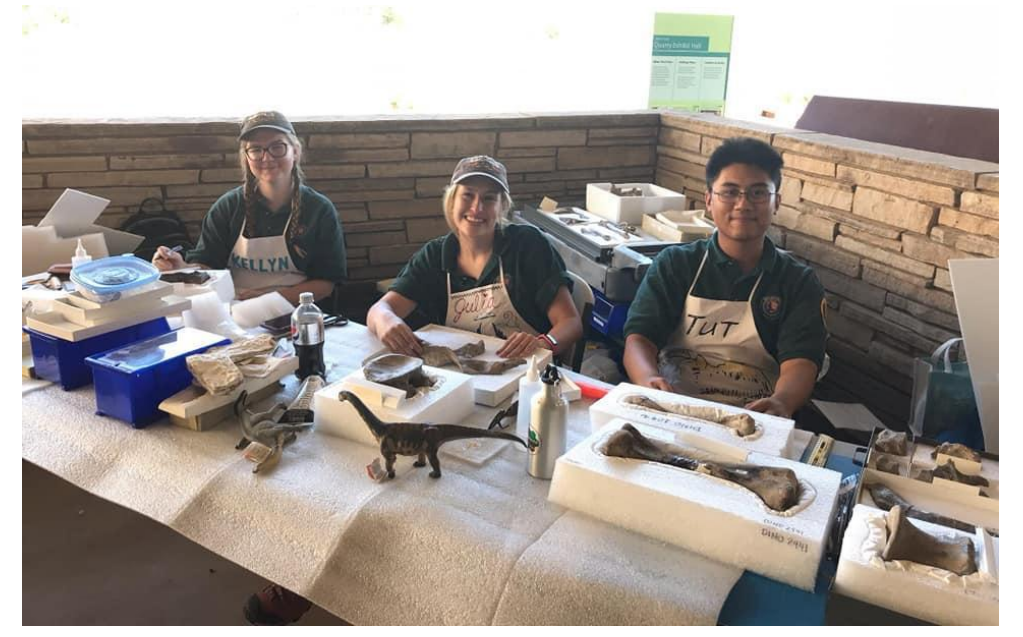

- Outreach E Education

○ Thur. \& Fri., 9:30 AM - 4:30 PM

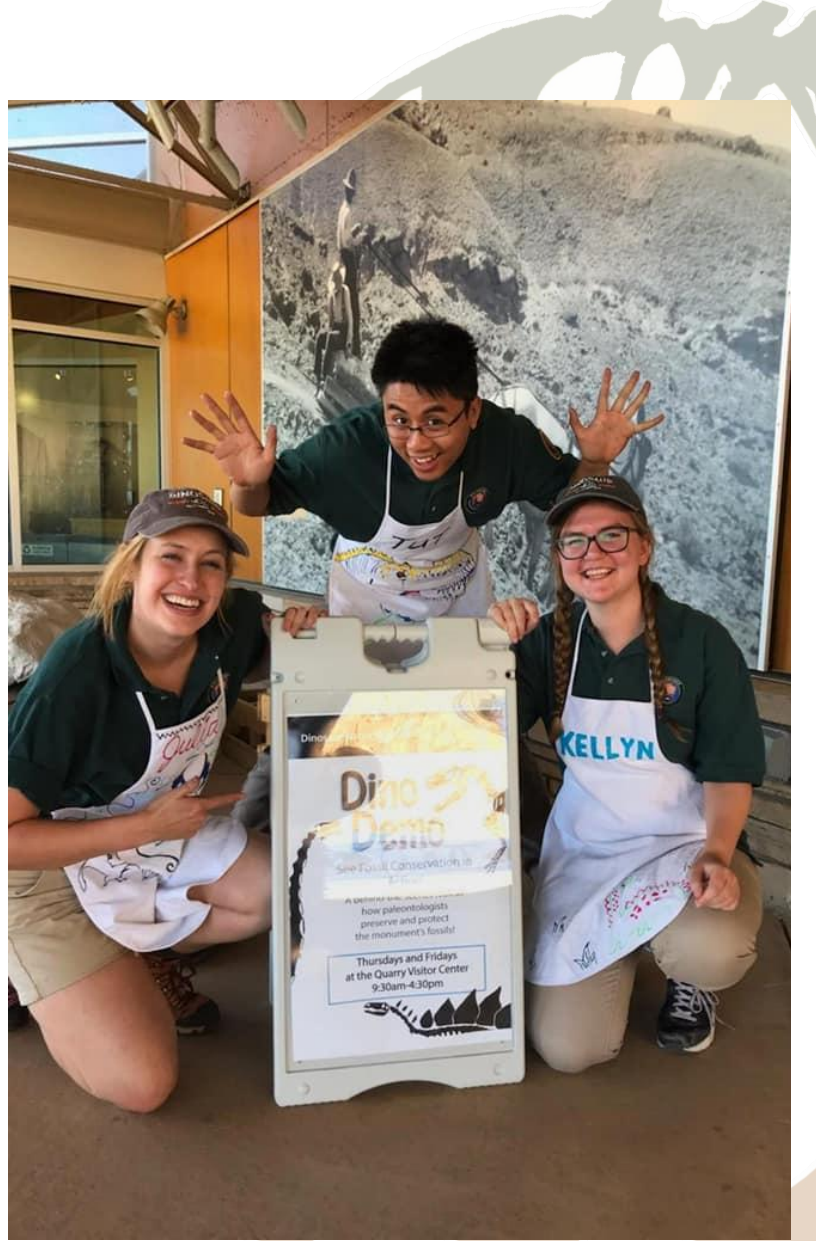

- Foam \& Plaster cradles

- 200-400+ visitors/day 


\section{Tackling the Issues}

- Outreach \& Education

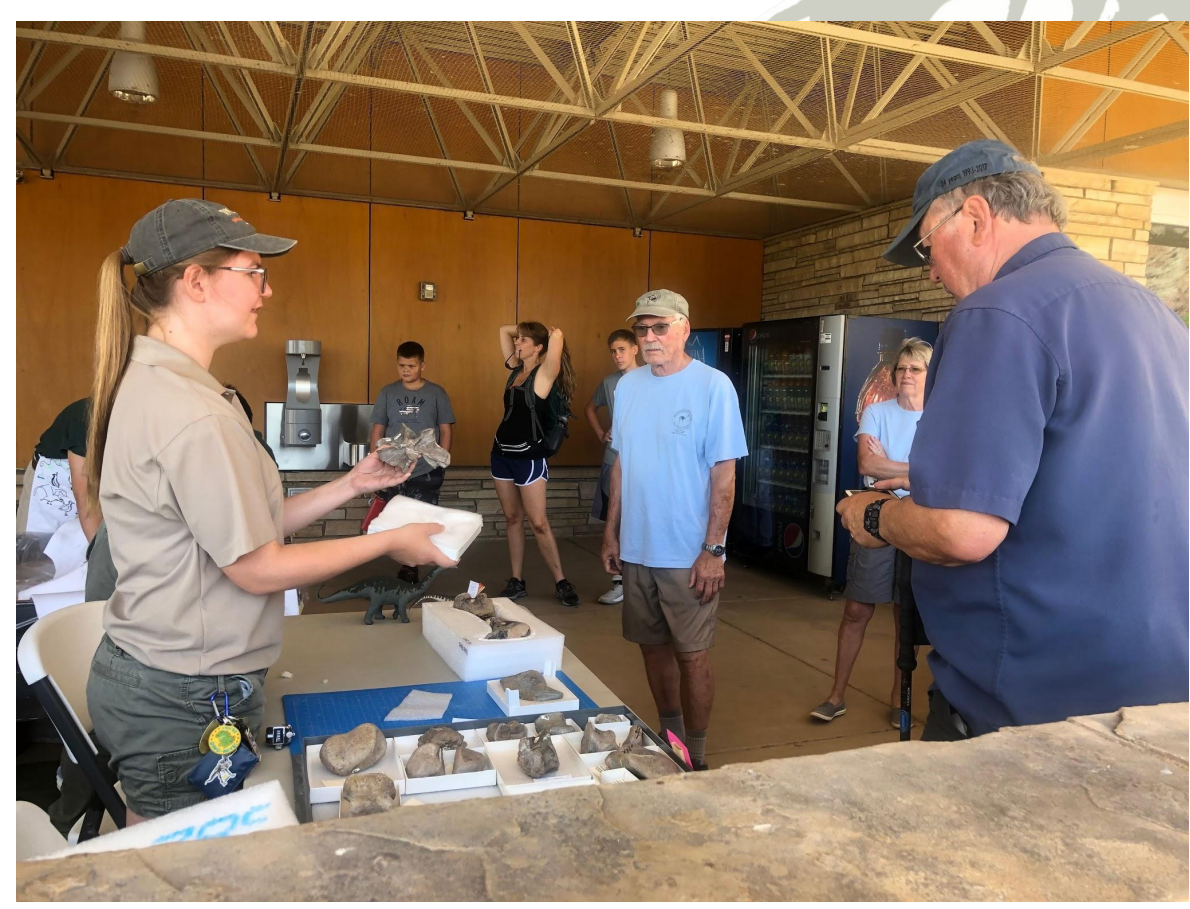

○ Thur. \& Fri., 9:30 AM - 4:30 PM

- Foam \& Plaster cradles

- 200-400+ visitors/day 


\section{Conclusions}

- Cradle-making was effective in stabilizing fossils

- Hantavirus mitigation practices will have to continue in future collections management at Canyon Visitor Center

- Outreach has impacted 3000+ people

- Laying foundations for ongoing collections management at DINO 


\section{What's Next?}

- Fossil stabilization efforts will need to continue

- Currently only short-term solution

- Long-term solution: erect a new collections facility

- New facility would need to simultaneously protect older specimens and accommodate new acquisitions 


\section{Special Thanks To:}

ReBecca Hunt-Foster

Mathew Wedel

Julia Anderson

Brian Engh

John Foster

Don DeBlieux

Rangers Ben, Nate, and Nik

Katie Nemmer

Adam Rountrey

Kelsey Ison

Steve Sroka
Ceological Söciety of America

Ceoscientists-in-the-Parks

Stewards Individual Placement

Program

Mosaics in Science Diversity Internship

Greening Youth Foundation

Environment for the Americas 